Anna Zingaro

Università di Bologna

https://doi.org/10.18778/8220-506-0.15

\title{
DAL DOLCE STIL NOVO ALLA DOLCE VITA: LA PERCEZIONE DELL'ITALIANITÀ IN UN LABORATORIO TEATRALE PER STRANIERI
}

Riassunto: In questo articolo si svilupperà una riflessione sulle potenzialità di un laboratorio di teatro in lingua italiana per stranieri ai fini dello sviluppo della competenza comunicativa e culturale, mettendo in luce in particolare la percezione dell'italianità da parte degli apprendenti. Si illustrerà come la costruzione dei personaggi, dei dialoghi e degli ambienti in cui far svolgere l'azione abbia contribuito a stimolare le capacità interpretative e immaginative degli apprendenti. Ciò ha permesso loro di esprimere la propria percezione della cultura italiana, che spazia dalla formazione letteraria e artistica fino ad includere il più ampio immaginario derivante dai mass media, in particolare dai social network, e dalla canzone italiana. Dopo aver illustrato la metodologia adottata nella gestione del laboratorio, verrà fornita una panoramica dei riferimenti culturali italiani inseriti nei testi teatrali messi in scena, prospettando anche uno sviluppo di tale prassi verso una sempre maggiore cooperazione tra pari nello sviluppo del testo teatrale.

Parole chiave: italiano L2, teatro, apprendimento cooperativo, immaginazione, cultura italiana.

\footnotetext{
Abstract: From Dolce Stil Novo to Dolce Vita: the perception of Italianness in a theater workshop for foreigners. This paper focuses on the potential of a theatre workshop to develop the communicative and cultural competence, the latter being related to the way Italianness is perceived by students of Italian as a Second Language. It first examines how a theatre workshop based on activities like constructing characters, dialogues and settings has proven useful in bringing into play the interpretive and imaginative skills of learners. Moreover, it shows how these activities allow learners to express their perception of the Italian culture through a mixture of both their literary and artistic education and a broader imaginary arising from the mass media, in particular from social networks and Italian songs. After illustrating the methodology adopted in the workshop, an overview of the Italian cultural references included in the theatrical texts staged will be provided, also envisaging a further development of this
} 
activity towards an ever greater cooperation between the students in the production of the theatrical text.

Keywords: Italian as a Second Language, theatre, cooperative learning, imagination, Italian culture.

\section{Introduzione}

In questo articolo si illustrerà il contributo dell'attività teatrale in lingua italiana all'acquisizione linguistica e allo sviluppo della competenza comunicativa e culturale da parte degli apprendenti stranieri, mettendo in luce in particolare la loro percezione dell'italianità ${ }^{1}$.

Le sezioni seguenti saranno incentrate sul contesto didattico in cui si è sviluppato il progetto e sulla drammaturgia per il primo triennio di attività del laboratorio (2017-2019). A questo proposito, si presenteranno i riferimenti culturali italiani a letteratura, arte, cinema, social network e televisione che sono stati inseriti nei testi teatrali prodotti, mostrando come il laboratorio abbia contribuito sia ad ampliare la cultura generale degli apprendenti, sia a far emergere la loro personale percezione dell' italianità.

\section{Contesto didattico}

Il laboratorio teatrale in lingua italiana per stranieri si è sviluppato presso il Dipartimento di Interpretazione e Traduzione (DIT) dell'Alma Mater Studiorum - Università di Bologna (Campus di Forlì), dove dal 1992 studenti e docenti collaborano all'organizzazione di laboratori teatrali in lingua straniera.

Si tratta di un'attività extracurriculare che arricchisce l'offerta formativa di un dipartimento per sua natura votato all'internazionalizzazione e che è coordinata dall'Associazione Universitaria Teatrale SSenzaLiMITi, il cui nome riprende la sigla SSLiMIT della ex Scuola Superiore di Lingue Moderne per Interpreti e Traduttori (divenuta dal 2012 il sopra menzionato DIT).

I singoli laboratori sono coordinati dai docenti e tutor delle diverse sezioni linguistiche o dagli studenti stessi, coprendo totalmente o parzialmente, a seconda delle adesioni ricevute, il ventaglio delle lingue insegnate presso

1 Il tema di questo articolo è stato parzialmente presentato al Convegno Internazionale "Immagini e immaginari della cultura italiana" Università degli Studi "Juraj Dobrila" di Pola (21-23 settembre 2017). In quell'occasione l'intervento era focalizzato solo sul primo anno di attività del laboratorio (cfr. Zingaro 2019). 
il Dipartimento, ossia: arabo, bulgaro, cinese, francese, giapponese, inglese, italiano L1, portoghese, russo, slovacco, spagnolo e tedesco.

L'obiettivo finale di tale attività è la Rassegna Babele Teatrale, organizzata dall'Associazione SSenzaLiMITi, che si svolge ogni anno presso il Teatro Comunale Diego Fabbri di Forlì, durante la quale vengono messi in scena gli spettacoli dei vari laboratori teatrali del Dipartimento.

Dall'A.A. 2016-2017 l'Associazione SSenzaLiMITi include anche il laboratorio in lingua italiana per stranieri, a cui saranno dedicate le sezioni successive.

\section{Organizzazione del laboratorio}

Sin dal suo primo anno di attività il laboratorio teatrale, coordinato dall'autrice del presente articolo in qualità di docente e tutor di Lingua Italiana L2, è stato caratterizzato da una grande eterogeneità dal punto di vista sia della provenienza geografica degli apprendenti (nello specifico: Austria, Canada, Cina, Ecuador, Francia, Giappone, Lituania, Regno Unito, Romania, Russia e Spagna), sia dei loro livelli di competenza, che spaziavano dal livello A2 ai livelli avanzati, fino ad includere anche alcuni apprendenti di madrelingua italiana. La presenza di questi ultimi è stata fondamentale per stimolare un confronto linguistico e culturale con gli apprendenti stranieri, fornendo un supporto linguistico globale relativamente alla pronuncia, l'intonazione, l'uso del lessico e le istruzioni per la messa in scena.

All'approccio didattico adottato per la gestione del laboratorio teatrale sarà dedicata la sezione successiva.

\section{Immaginazione e cooperazione}

Per sfruttare al meglio le potenzialità dell'attività teatrale nel favorire l'acquisizione linguistica e l'approccio con la cultura, l'obiettivo principale di questo laboratorio è stato l'elaborazione di testi basati su situazioni della vita quotidiana e con frequenti riferimenti alla cultura italiana.

Quanto affermato rispetto all'elaborazione di una trama, e non alla sola messa in scena, lascia intendere la decisione di non adottare un testo teatrale già esistente, ma di scriverne uno ex novo. Si tratta di una scelta compiuta con l'obiettivo ben preciso di promuovere attraverso il teatro una didattica pragmatica il più vicina possibile ai reali obiettivi comunicativi dei discenti (Balboni 2003). 
La stesura del testo teatrale si svolge in due fasi. Innanzitutto, la prima bozza viene prodotta autonomamente dalla docente-coordinatrice del laboratorio, con la successiva rilettura e collaborazione da parte di alcuni membri del gruppo italofoni nativi. Questa bozza comprende l'impostazione di alcune scene già complete in tutti i loro elementi (personaggi in scena, con le relative battute e le istruzioni di regia) e di altre lasciate volutamente incomplete, allo scopo di richiedere nella seconda fase del lavoro la collaborazione degli altri membri del gruppo al completamento delle parti mancanti del testo e, dunque, della trama stessa. Inoltre, i partecipanti al laboratorio hanno la possibilità di proporre delle modifiche anche alle parti del testo teatrale già complete, scegliendo liberamente il ruolo da interpretare tra ruoli principali, secondari e comparse.

Si tratta, dunque, di un approccio più flessibile rispetto a quanto suggerito da Cangià (1998: 207-227) circa l'imposizione di un copione da parte del docente: coinvolgere i partecipanti al laboratorio teatrale nel completamento e/o nella modifica di un canovaccio iniziale prodotto dalla docente consente a tutti di contribuire al risultato finale utilizzando le tecniche del role-play e dell'improvvisazione sulla base degli studi sull'efficacia della sfera ludica nell'insegnamento delle lingue ${ }^{2}$.

Questa scelta operativa, che ha lo scopo di far familiarizzare gli apprendenti con la struttura del testo teatrale, favorirne il coinvolgimento e stimolarne la motivazione, rientra nella prassi didattica di tipo cooperativo, le cui basi teoriche si sono sviluppate negli Stati Uniti all'inizio del Ventesimo secolo tramite la filosofia educativa del pedagogista John Dewey e la psicologia sociale dello psicologo Kurt Lewin, che si fondano sulla necessità e sul valore dell'interazione e della cooperazione nell'ambito scolastico. All'interno della classe l'apprendimento avviene in piccoli gruppi tramite ruoli e compiti basati sull'interdipendenza positiva (cfr. Johnson e Johnson 1989), ossia il raggiungimento di un obiettivo è ottenuto tramite la cooperazione tra i membri del gruppo, che si aiutano a vicenda sentendosi corresponsabili del reciproco percorso, poiché «l'efficacia complessiva del gruppo è superiore alla somma di quella delle sue parti» (Johnson, Johnson e Holubec 2001: 23).

Nello specifico del laboratorio teatrale, lavorare sulle singole scene ha permesso ai partecipanti di concentrarsi sugli atti linguistici richiesti nelle diverse situazioni comunicative e sugli scopi pragmatici da raggiungere ${ }^{3}$. Ad esempio, nel caso in cui un personaggio debba invitare un altro ad uscire, viene

2 Per un approfondimento su questo tema si rimanda a: Lozanov e Gateva (1983); Caon e Rutka (2003); Begotti (2006); Mezzadri (2002); Mollica (2010); Oliva (1999); Sisti (2000) e Sudati (2013).

3 Per un approfondimento su questo tema si rimanda a: Ardissino (2010); Ardissino e Stroppa (2001); Ardissino e Stroppa (2009). 
chiesto ai partecipanti di elencare le espressioni linguistiche con cui è possibile accettare/rifiutare con (s)cortesia l'invito, formulare una nuova proposta, insistere, accettare o declinare di nuovo ecc. Pertanto, è risultata fondamentale la presenza nel gruppo di membri di italofoni nativi, oltre alla stessa docente, che potessero guidare gli apprendenti nella scelta delle espressioni linguistiche più appropriate rispetto alla situazione comunicativa, al suo registro linguistico formale o informale e agli scopi pragmatici coinvolti.

Tutto ciò si è svolto nel contesto di un'attività che già per sé favorisce l'acquisizione linguistica, poiché, se da un lato la ripetizione e memorizzazione delle battute del testo teatrale favoriscono l'acquisizione passiva di nuove strutture linguistiche, dall'altro l'immedesimazione in un personaggio a cui "delegare" la produzione orale e la relativa possibilità di sbagliare, consentono di abbassare il livello del filtro affettivo (Krashen 1985) e aumentare la fiducia in sé.

Chiarita l'impostazione operativa data al laboratorio, la sezione successiva sarà dedicata ad alcuni riferimenti culturali italiani a letteratura, arte, cinema, social network e televisione che sono stati inseriti nei testi teatrali messi in scena.

\section{La percezione della cultura italiana}

L'obiettivo generale del laboratorio è la realizzazione di commedie in cui citazioni colte letterarie e artistiche si intreccino a riferimenti a pubblicità, programmi televisivi, musica pop e fenomeni di costume all'interno di trame ironiche e, a tratti, dissacranti.

Gli elementi culturali italiani da inserire nei testi vengono inizialmente proposti dalla docente-coordinatrice del laboratorio e in una seconda fase si svolge un confronto diretto con i membri del gruppo, finalizzato alla selezione definitiva.

Questa collaborazione tra la docente e gli apprendenti ha portato alla produzione di tre brevi commedie che verranno di seguito illustrate.

\section{a. Letteratura italiana}

Nella commedia Come un libro aperto (2017) uno studente in Erasmus in Italia, nel tentativo di fare colpo sulla ragazza italiana di cui è innamorato, prende in prestito un libro di poesie, per poi scoprire che si tratta di un libro magico: ogni volta che lo apre, al suo fianco si materializzano Dante Alighieri, Giacomo Leopardi e Gabriele D’Annunzio, pronti a dargli consigli in ambito sentimentale, ciascuno secondo il proprio vissuto e il proprio stile letterario.

È stata adottata una chiave ironica nella costruzione dei personaggi degli autori, che sono diventati tre figure stereotipate (romantico e solenne Dante, introverso ed imbranato Leopardi e infine D’Annunzio, il seduttore 
egocentrico), le cui vite e opere letterarie hanno subito una trasposizione in chiave moderna, con l'attribuzione di alcuni comportamenti e oggetti tipici della generazione dei Millennials. Gli autori, infatti, sono stati rappresentati come tre giovani amici che vivono nel Mondo della Letteratura, una sorta di realtà parallela, dove la loro vita e produzione letteraria si affianca all'uso di cellulari, internet e social network.

Chiaramente un simile lavoro di trasposizione presuppone la conoscenza da parte degli apprendenti della biografia e delle opere principali degli autori, che non sempre rientrano tra le conoscenze pregresse maturate nei percorsi di studio svolti nei loro paesi di origine. Pertanto, allo scopo di far familiarizzare gli apprendenti con i tre autori, nelle lezioni di lingua italiana L2 sono state programmate attività di lettura e comprensione testuale di brani sulla vita degli autori, con annesse attività di completamento del testo con parole mancanti o riordino dei paragrafi per ricostruirne la struttura originaria. Inoltre, sono stati sempre proposti brevi estratti delle loro opere principali, corredati da una breve sinossi e presentati sempre con una traduzione a fronte in inglese, una scelta, quest'ultima, basata unicamente su ragioni di praticità, essendo l'inglese l'unica lingua comune a tutti i membri del gruppo oltre all'italiano. Nelle ore extracurriculari ciascuno poteva, eventualmente, reperire le traduzioni nella propria L1 delle opere trattate.

Un'ulteriore strategia messa in atto per facilitare l'approccio con gli autori della letteratura è stata la consultazione della pagina Facebook Se i social network fossero sempre esistiti, ove si ipotizza con ironia come sarebbe stata la vita di personaggi celebri di tutte le epoche (storici, della letteratura, dell'arte, della musica, ecc.), se avessero avuto a disposizione gli attuali mezzi di comunicazione. La lettura delle ipotetiche chat tra personaggi celebri presenti sulla pagina ha fornito l'ispirazione per l'ironia e il registro linguistico colloquiale da utilizzare nei dialoghi. Nello specifico, la pagina ha ispirato la scelta dei tre autori, la trasposizione in chiave moderna di alcuni loro comportamenti (ad esempio, l'uso del cellulare, le cui suonerie, come vedremo di seguito, sono state scelte appositamente per creare dei riferimenti ironici alle loro personalità, eccetera) e l'adozione di una prospettiva volutamente non cronologica nel rappresentare degli autori distanti tra loro nel tempo come tre amici.

Pertanto, Dante è stato rappresentato come il romantico ed idealista del trio degli amici-autori, che ogni giorno invia sonetti a Beatrice, la quale, però, non solo «visualizza e non risponde», ma «altrui saluta». Queste due azioni, che si riferiscono, rispettivamente, al ghosting, ossia l'atteggiamento di chi visualizza sullo schermo i messaggi ricevuti in una chat, senza però rispondere, e al secondo verso del sonetto Tanto gentile e tanto onesta pare de La Vita Nuova, costituiscono una rivisitazione in chiave ironica della figura della donna-angelo, destinataria dell'amore stilnovistico. 
D’Annunzio, vanitoso, sicuro di sé e del suo potere seduttivo, si distingue per le frequenti battute a doppio senso, con le quali entra costantemente in contrasto con Leopardi, un giovane introverso che invece trascorre il sabato sera nella piazzetta del villaggio (un chiaro riferimento alla poesia Il sabato del villaggio) ed è impacciato con le donne, che non rispondono mai ai suoi messaggi.

Le loro interazioni hanno frequenti riferimenti ironici alle loro opere letterarie, di cui si riportano alcuni esempi:

- Gabry: «Amici, ieri sera sono stato ad una festa: la distruzione totale!»

- Dante: «E come sempre la diritta via era smarrita!»

ove la frase di Dante contiene una citazione parziale del terzo verso del primo canto dell'Inferno;

- Jack: «Sabato allora tutti in piazzetta da me a Recanati, per il sabato del villaggio?»

- Gabry: «No, piuttosto vado a prendermi la peste con Alex Manzoni!»

In questo scambio di battute sono stati inseriti riferimenti sia alla poesia Il sabato del villaggio di Leopardi, con l'ulteriore rimando geografico al noto luogo di origine del poeta, sia ai capitoli XXXI e XXXII dedicati alla peste nel romanzo I Promessi Sposi di Alessandro Manzoni, il cui nome è stato abbreviato in Alex, nell'ottica della trasposizione in chiave moderna dei personaggi citati (come avvenuto anche per Leopardi e D’Annunzio, divenuti rispettivamente Jack e Gabry);

- Dante: «Nel mezzo del cammin mi ritrovai bloccato da uno sciopero inaspettato. Ahi, serva Trenitalia!»

In questo caso vi sono due citazioni tratte dalla Divina Commedia: $\ll \mathrm{Nel}$ mezzo del cammin di nostra vita» (Dante 1968: 4) (Inferno canto I, verso 1) e «Ahi serva Italia, di dolore ostello» (Dante 1968: 65) (Purgatorio, canto VI, verso 76), nel quale si innesta un riferimento ironico ai ritardi dei treni del sistema ferroviario nazionale italiano.

Come affermato in precedenza, gli elementi tratti dalla letteratura italiana non sono gli unici riferimenti culturali inseriti nel testo teatrale. Sono presenti anche richiami a pubblicità, programmi televisivi e fenomeni di costume, che sono stati suggeriti dai membri del gruppo in quanto percepiti come rappresentativi della cultura di massa italiana e hanno subito un riadattamento funzionale al loro inserimento nella trama. Si citano a titolo esemplificativo i seguenti elementi: le canzoni Andiamo a comandare di Fabio Rovazzi e Non è l'inferno di Emma Marrone sono diventate le suonerie dei rispettivi cellulari di D’Annunzio e Dante, segnando la loro entrata in scena in uno degli atti della commedia; il concorso di Miss Italia è stato trasposto nel concorso di Miss Donzelletta che Vien dalla Campagna, con un richiamo al primo verso de Il Sabato del Villaggio di Leopardi («La donzelletta vien dalla campagna») 
(Leopardi 1997: 167); il quotidiano locale online «Forlì Today» è diventato il «Recanati Oggi», ossia un immaginario quotidiano letto da Leopardi; la fiera del fumetto Lucca Comics, nota meta toscana per cosplayer e appassionati di fumetti, vede tra i partecipanti anche D'Annunzio, vestito da Superuomo presso lo stand della casa editrice Marvel Comics, nota per le pubblicazioni dedicate in particolare ai supereroi.

Per la caratterizzazione del personaggio di Dante è stato, poi, utilizzato un rotolo di carta igienica tenuto in mano come fosse una pergamena, un'idea tratta da uno spot televisivo di una nota marca di carta igienica, nel quale, per esaltare la lunghezza del prodotto, viene rappresentato Dante che ne utilizza un rotolo per scrivere la Commedia. Pur non potendo citare la marca del prodotto nello spettacolo, il pubblico ha colto con ilarità il nesso con lo spot in questione.

\section{b. La storia dell'arte}

Nel 2018 è stata realizzata una commedia intitolata Togli l'arte e mettila da parte, nella quale alcune celebri opere d'arte rubate si risvegliano in un magazzino e progettano la fuga con l'aiuto di due giovani che si sono accorti della loro presenza.

Le opere d'arte italiane inserite nel testo sono la Gioconda e la Dama con l'ermellino di Leonardo da Vinci ed Ebe di Antonio Canova, a cui si aggiungono opere di altri paesi, quali l'Urlo di Edvard Munch, un autoritratto di Van Gogh, la Ragazza dall'Orecchino di Perla di Jan Vermeer e una mummia egizia.

Circa la metodologia adottata, l'approccio con le opere d'arte inserite nel testo teatrale si è svolto in due fasi. Innanzitutto, è stato svolto un approfondimento di tipo iconografico, chiedendo a ciascuno di raccogliere informazioni sull'opera d'arte da interpretare, l'autore e il relativo periodo storico, consultando manuali di storia dell'arte e pagine web dedicate. A supporto dellanalisi iconografica sono stati utilizzati anche i testi di Andriuzzi (2017), Angelino, Ballarin e Balboni (2006), Mantovano (1996) e Peruzzi (2010). La ricerca di immagini delle opere d'arte è stata utile sia a raggiungere la maggior verosimiglianza possibile tramite la scelta di costumi, trucco e posture, sia a valutarne le diverse versioni esistenti e sceglierne una, come nel caso delle 4 versioni di Ebe realizzate da Canova tra il 1796 e il 1817, tra cui è stata selezionata quella custodita ai Musei San Domenico di Forlì, e dei 37 autoritratti di Van Gogh esistenti.

Anche in questo caso è stata consultata una pagina Facebook, ossia Se $i$ quadri potessero parlare, ove ai personaggi raffigurati nelle opere d'arte di tutte le epoche vengono attribuite didascalie o battute comiche in romanesco. La pagina ha fornito, anche in questo caso, l'ispirazione per l'attribuzione di comportamenti umani alle opere d'arte, adeguati alla loro caratterizzazione, per l'ironia e il registro linguistico colloquiale da utilizzare nella creazione dei 
dialoghi. Ad esempio, i dialoghi tra la Dama con l'ermellino e la Gioconda sono caratterizzati da frequenti scontri in cui ciascuna ritiene di essere l'opera meglio riuscita a Leonardo da Vinci, mentre invece Ebe, data la sua raffigurazione con una brocca di bronzo, è stata rappresentata come una giovane allegra che beve un noto vino romagnolo, allo scopo di inserire un riferimento culturale locale.

A questo proposito, la sezione successiva si focalizzerà proprio sulla rivisitazione in chiave romagnola di un classico del cinema degli anni Cinquanta del Novecento.

\section{c. La Dolce Vita ... romagnola}

Nel suo terzo anno di attività (2019) il laboratorio ha messo in scena una rivisitazione del film Vacanze Romane (Roman Holiday) di William Wyler in uno spettacolo dal titolo Vacanze Romagnole. La trasposizione ai giorni nostri e nella città di Forlì ha richiesto un lavoro di selezione dei riferimenti turistici e culturali romagnoli da sostituire a quelli originari romani.

Nella pellicola la principessa Anna di un imprecisato stato europeo, arrivata in visita ufficiale a Roma, si sottrae alla sorveglianza dei dignitari e si immerge in incognito nella Dolce Vita della città, accompagnata dal giornalista Joe Bradley, che, mirando ad uno scoop, a sua volta non le svela la sua identità. Nella trasposizione teatrale, allo scopo di inserire dei riferimenti culturali italiani che si distaccassero dall'originale, la protagonista è diventata l'immaginaria principessa Sofia di Valle d'Aosta, erede della ex casa reale italiana dei Savoia, il cui titolo è stato inventato come riferimento al ramo cadetto dei SavoiaAosta. Per familiarizzare con la storia dell'ultimo re d'Italia e dei suoi eredi sono state consultate delle pagine web sul tema tratte da Wikipedia, al fine di fornire riferimenti storici in testi che non risultassero troppo complessi rispetto al livello di conoscenza e competenza linguistica dei partecipanti.

Arrivata con la madre e la sorella in Italia in vista di un matrimonio vantaggioso con un ricco industriale romagnolo, la principessa Sofia sfugge alla sorveglianza della famiglia e si imbatte in un giovane stagista del quotidiano L'Urlo di Romagna (una parodia del quotidiano La Voce di Romagna), che inizialmente vorrebbe sfruttarla per uno scoop che lo salvi dal licenziamento.

Data questa impostazione alla trama, è stato poi necessario un lavoro cooperativo all'interno del gruppo per sostituire i luoghi turistici romani mostrati nel film con alcune località romagnole (Bertinoro, Cesena, Cervia, Ravenna e Riccione) e con alcuni luoghi caratteristici di Forlì (es. Piazza Saffi, l'abazia di San Mercuriale e i giardini pubblici). L'adattamento ha, infine, implicato anche l'inserimento di prodotti e abitudini locali (l'acquisto della piadina romagnola al relativo chiosco a conduzione familiare, la passeggiata al Parco Urbano ecc.). Tutto ciò ha portato alla trasposizione, in chiave locale ma soprattutto parodistica, della Dolce Vita romana nella Dolce Vita romagnola, 
che ha permesso ai partecipanti al laboratorio di familiarizzare sia con alcuni riferimenti noti sul piano nazionale (la monarchia, l'ultimo re d'Italia e i suoi eredi), sia con luoghi e tradizioni della Romagna, rafforzando così il legame che normalmente si instaura tra dei giovani provenienti da vari paesi europei e la sede in cui scelgono di svolgere il progetto Erasmus.

\section{d. Musica}

In ciascuno degli spettacoli è stato dato ampio spazio, infine, alla musica italiana. La scelta dei brani è stata fatta tramite associazioni di idee tra il contenuto delle singole scene e le conoscenze dei membri del gruppo in ambito musicale (ad esempio: quali brani musicali italiani vi vengono in mente che trasmettono gioia, angoscia, pericolo, tristezza, ecc.?), senza porre alcun vincolo circa i generi musicali o il periodo storico. Dopo l'ascolto dei brani, le diverse proposte sono state votate dai membri del gruppo, portando alla selezione di alcune canzoni italiane che spaziano dagli anni Settanta del Novecento agli anni Dieci del Duemila.

\section{Conclusione}

Per poter favorire l'acquisizione di una lingua, è necessario che i docenti creino situazioni che coinvolgano e stimolino gli apprendenti e attività che consentano uno sviluppo a tutto tondo della competenza comunicativa e culturale. Come illustrato precedentemente, l'attività teatrale in sé consente di acquisire nuove strutture linguistiche attraverso la memorizzazione delle battute, di abbattere i filtri affettivi ed aumentare la fiducia in sé interpretando un ruolo, oltre che di coinvolgere anche la motricità e la sensorialità. Nel caso specifico, l'inserimento nel testo teatrale di elementi culturali italiani, frutto di una raccolta di idee cooperativa all'interno del gruppo, ha da un lato facilitato l'approccio da parte degli apprendenti con la cultura italiana, e dallaltro ha permesso loro di mettere in scena la loro percezione dell'italianità, con riferimenti a letteratura, arte, cinema, social network, televisione e musica.

Inoltre, la scelta di dare un'impostazione cooperativa alla produzione del testo teatrale ha permesso di creare e rafforzare i rapporti interpersonali tra gli apprendenti e di potenziarne le capacità immaginative e interpretative. La partecipazione e il gradimento mostrato dagli apprendenti e dal pubblico che ha assistito alle rappresentazioni teatrali nell'arco dei 3 anni danno conferma della riuscita delle attività proposte. Si prospetta, pertanto, uno sviluppo di tale prassi nel prossimo anno accademico, focalizzando maggiormente le attività del laboratorio teatrale sulla valenza della cooperazione tra pari nel completamento del testo teatrale e nella ricerca di elementi culturali da inserire. 


\section{Bibliografia}

Alighieri, Dante (2a ed., 1968 [1955]). La Divina Commedia, I Inferno, [in] Natalino Sapegno (a c. di), Firenze, La Nuova Italia.

Alighieri, Dante (2a ed., 1968 [1955]). La Divina Commedia, II Purgatorio, [in] Natalino Sapegno (a c. di), Firenze, La Nuova Italia.

Andriuzzi, Rossana (2017). L'italiano dell'arte. Corso di lingua italiana, Milano, Hoepli.

Angelino, Maddalena, Ballarin, Elena e Balboni, Paolo Ernesto (a c. di) (2006). L'italiano attraverso la storia dell'arte, Perugia, Guerra Edizioni.

Ardissino, Erminia (2010). "Imparare l'italiano L2/LS con testi teatrali", Italiano linguadue (2) 1, pp. 111-123.

Ardissino, Erminia e Stroppa, Sabrina (2001). Leggere testi letterari, Milano, Paravia - Mondadori.

Ardissino, Erminia e Stroppa, Sabrina (2009). La letteratura nei corsi di lingua. Dalla lettura alla creatività, Perugia, Guerra Edizioni.

Balboni, Paolo, Ernesto (2003). Le sfide di Babele. Insegnare le lingue nelle società complesse, Torino, Utet.

Begotti, Paola (2006). L'insegnamento dell'Italiano ad adulti stranieri, Perugia, Guerra Edizioni. Cangià, Caterina (1998). L'altra glottodidattica, Firenze, Giunti.

Caon, Fabio e Rutka, Sonia (2003). La lingua in gioco, Perugia, Guerra Edizioni.

Johnson, David, W. e Johnson, Roger, T. (1989). Cooperation and Competition: Theory and Research, Edina, MN, Interaction Books.

Johnson, David, W., Johnson, Roger, T. E Holubec, Edythe (2001). Apprendimento cooperativo in classe. Migliorare il clima emotivo e il rendimento, trad. it Lucio Marinelli, Trento, Erickson (ed. orig. Cooperative learning in the classroom, Alexandria, VA, ASCD Publications).

Krashen, Stephen D. (1985). The Input Hypothesis: Issues and Implications, London, Longman. Leopardi, Giacomo (4a ed., 2014 [1997]). Tutte le poesie, tutte le prose e lo Zibaldone, [in] Lucio Felici e Emanuele Trevi (a c. di), Roma, Newton Compton.

Lozanov, Georgi e Gateva, Evelina (1983). Metodo Suggestopedico per l'insegnamento delle lingue straniere, trad. it. Simeon Todorov, Roma, Bulzoni Editore (ed. orig. Сугестопедично практическо рбководство за преподаватели по чужди езици, Sofia, Научноизследователски институт по сугестология, 1981).

Mantovano, Francesca (1996). L'italiano dell'arte, Perugia, Guerra Edizioni.

Mezzadri, Marco (2002). “Imparare giocando”, In.it, (3) 3, Perugia, Guerra Edizioni.

Mollica, Anthony (2010). Ludolinguistica e glottodidattica, Perugia, Guerra Edizioni.

Oliva, Gaetano (1999). Il teatro nella scuola. Aspetti educativi e didattici, Milano, LED.

Peruzzi, Paola (2010). "L'uso didattico dell'immagine", [in] Pierangela Diadori (a c. di), Insegnare italiano a stranieri, Milano, Mondadori, pp. 274-287.

Sisti, Flora (2000). Lo sviluppo delle abilità orali attraverso la drammatizzazione, Trieste, Edizioni Goliardiche.

Sudati, Ilaria (2013). "La didattica ludica. Teoria e applicazioni pratiche nell'insegnamento dell'italiano L2 ad adulti”, Italiano LinguaDue, (2), pp. 211- 225.

Zingaro, Anna (2019). "L'immaginario della cultura italiana attraverso un laboratorio teatrale per stranieri”, [in] Eliana Moscarda Mirković e Tanja Habrle (a c. di), Sguardo 
sull'immaginario italiano. Aspetti linguistici, letterari e culturali Pola, Università degli Studi 'Juraj Dobrila' di Pola (Croazia) - Facoltà di Studi Interdisciplinari, Italiani e Culturali - Dipartimento di Studi Italiani, pp. 157-173.

\section{Sitografia}

www.facebook.com/seiquadripotesseroparlare/ [18/02/2020].

www.facebook.com/seisocialnetwork/ [18/02/2020]. 